\title{
Status of three-neutrino mixing
}

\author{
Francesco Capozzi* \\ Dipartimento di Fisica e Astronomia "Galileo Galilei," Via Marzolo 8, 35131 Padova, Italy. \\ Istituto Nazionale di Fisica Nucleare - Sezione di Padova, Via Marzolo 8, 35131 Padova, Italy. \\ E-mail: francesco.capozzi@pd.infn.it

\section{E. Lisi}

Istituto Nazionale di Fisica Nucleare, Sezione di Bari, Via E. Orabona 4, I-70126 Bari, Italy

\author{
A. Marrone, A. Palazzo \\ Dipartimento Interateneo di Fisica “Michelangelo Merlin”, Università di Bari, Via G. \\ Amendola 173, I-70126 Bari, Italy \\ Istituto Nazionale di Fisica Nucleare, Sezione di Bari, Via E. Orabona 4, I-70126 Bari, Italy \\ D. Montanino \\ Dipartimento di Matematica e Fisica “Ennio de Giorgi”, Università del Salento, Via Arnesano, \\ I-73100 Lecce, Italy \\ Istituto Nazionale di Fisica Nucleare (INFN), Sezione di Lecce, Via Arnesano, I-73100 Lecce, \\ Italy
}

\begin{abstract}
We present an up-to-date global analysis of neutrino oscillation data in the standard three-neutrino framework, focusing on the status of the currently unknown parameters: the mass hierarchy, the octant of $\theta_{23}$ and the CP phase $\delta$. To our previous analysis we add the latest result from $\mathrm{T} 2 \mathrm{~K}$, both appearance and disappearance channels of $v$ and $\bar{v}$ data, NOvA, both appearance and disappearance channels of $v$ data, SBL reactor (Daya Bay and RENO) and updated atmospheric neutrino experiments (Super-Kamiokande).
\end{abstract}

Neutrino Oscillation Workshop

4 - 11 September, 2016

Otranto (Lecce, Italy)

${ }^{*}$ Speaker. 


\section{Introduction}

Almost all observations performed at neutrino oscillation experiments can be explained by the three-neutrino paradigm [1], where the flavor eigenstates $v_{\alpha}(\alpha=e, \mu, \tau)$ are a superposition of the mass eigestates $m_{i}(i=1,2,3)$ through the three mixing angles $\left(\theta_{12}, \theta_{13}, \theta_{23}\right)$ and a CP-violating phase $\delta$. However, neutrino oscillations depend on the neutrino masses $m_{i}$ through two quantities that we define as $\delta m^{2}=m_{2}^{2}-m_{1}^{2}>0$ and $\Delta m^{2}=m_{3}^{2}-\left(m_{2}^{2}+m_{1}^{2}\right) / 2$. According to the sign of $\Delta m^{2}$ we can distinguish two possible ordering of the mass eigenstates: the normal hierarchy $(\mathrm{NH})$, when $\Delta m^{2}>0$, and the inverted hierarchy (IH) in the opposite case. The two "solar" parameters $\left(\delta m^{2}, \theta_{12}\right)$ have been measured by solar experiments in combination with KamLAND, $\theta_{13}$ by shortbaseline experiments (SBL) and the "atmospheric" parameters $\left(\Delta m^{2}, \theta_{23}\right)$ by atmospheric and longbaseline (LBL Acc) experiments. However, it is still unknown if the mixing angle $\theta_{23}$ is close to maximal or not, and in this second case, which is its octant. Moreover, the value of $\delta$ is also still unknown, even though very recent data begin to constrain its allowed range at 2-3 $\sigma$ level. Finally, current experiments are only poorly sensitive to the hierarchy discrimination. In such a context, global analyses are a very useful tool to test the consistency of all available oscillation data and to obtain information about parameters that are not well constrained by a single class of experiments. In this contribution we present the results of a recent global analysis of oscillation data (as of summer 2016). In particular, with respect to our previous analysis [2], we add the latest result from T2K [3], both appearance and disappearance channels of $v$ and $\bar{v}$ data, NOvA [4], both appearance and disappearance channels of $v$ data, SBL reactor experiments (Daya Bay and RENO) $[5,6]$ and Super-Kamiokande (atmospheric neutrino data) [7].

\section{Constraints on single oscillation parameters}

We start the discussion on the results of the global analysis by considering one dimensional projections of $\Delta \chi^{2}=\chi^{2}-\chi_{\text {min }}^{2}$ on each parameter, where $\chi_{\text {min }}^{2}$ is the minimum value of the $\chi^{2}$ obtained by marginalizing over all the six oscillation parameters. The results are reported in Fig. 1, showing the confidence intervals, in terms of $\mathrm{N} \sigma$, for each oscillation parameter and for both $\mathrm{NH}$ (blue) and IH (red). Note that for the solar parameters, there is essentially no difference between the mass orderings, because the tiny effects related to NH-IH differences are unobservable in the fit. In this case only the curve for $\mathrm{NH}$ is reported. If we define the average $1 \sigma$ error as $1 / 6$ of the $\pm 3 \sigma$ range, our global fit implies the following fractional uncertainties: $\delta m^{2}(2.4 \%), \sin ^{2} \theta_{12}$, (5.8\%) $\Delta m^{2}(1.6 \%), \sin ^{2} \theta_{13}(3.9 \%)$ and $\sin ^{2} \theta_{23}$ (8.3\%). Moreover, at present the global analysis prefers the normal hierarchy with $\chi_{I H, \text { min }}^{2}-\chi_{N H, \min }^{2} \simeq 3.6$. In the following part of this section we discuss the main features of the global analysis, regarding both $\delta$ and $\theta_{23}$.

With respect to the CP-conserving case $\sin \delta=0$, values of $\sin \delta<0$ are expected to produce a slight increase (decrease) of events in the $v_{\mu} \rightarrow v_{e}$ oscillations for $\theta_{13}$ fixed (by reactors), consistently with the appearance results of $\mathrm{T} 2 \mathrm{~K}$ and in NOvA, although within large statistical uncertainties. This trend for $\delta$ is clearly confirmed by the results in Fig. 1, which show a best fit for $\delta \simeq 1.3-1.4 \pi$ in both $\mathrm{NH}$ and $\mathrm{IH}$, while opposite values around $\delta=0.5 \pi$ are disfavored at more than $3 \sigma$. Moreover, the CP-conserving values $\delta=0, \pi$, are disfavoured at $\sim 1.6 \sigma$, which corresponds to $90 \%$ confidence level for 1 degree of freedom. 

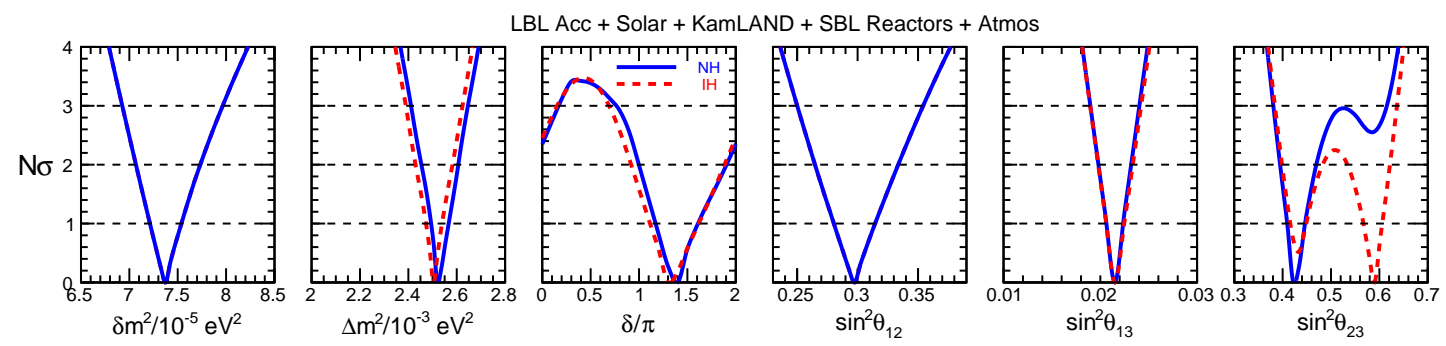

Figure 1: Bounds on the mass-mixing parameters from the combination of all neutrino oscillation experiments in terms of standard deviations $\mathrm{N} \sigma$ from the best fit. The solid lines (dashed) lines refer to $\mathrm{NH}(\mathrm{IH})$. Bounds on $\left(\delta m^{2}, \theta_{12}\right)$ are hierarchy-independent. Horizontal dotted lines mark the 1,2 , and $3 \sigma$ levels for each parameter

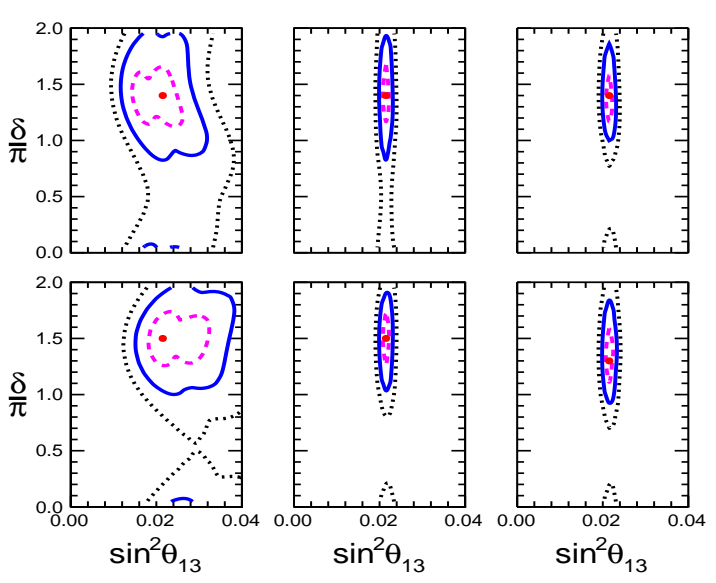

(a) Covariance plot for $\left(\sin ^{2} \theta_{13}, \delta\right)$ parameters

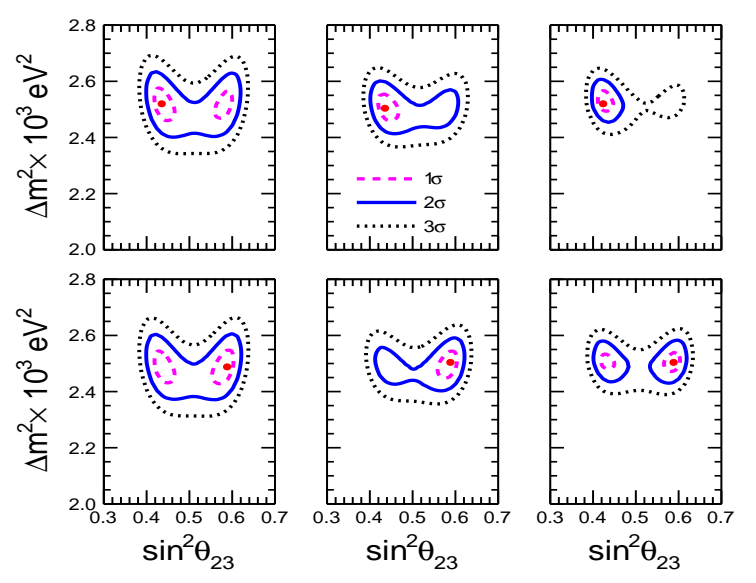

(b) Covariance plot for $\left(\sin ^{2} \theta_{23}, \Delta m^{2}\right)$ parameters

Figure 2: From left to right, the regions allowed at $N \sigma=1,2,3 \sigma$ refer to the analysis of LBL Acc+Solar+KL data (left panels), plus SBL reactor data (middle panels), plus Atmospheric data (right panels), with best fits marked by dots. The three upper (lower) panels refer to NH (IH).

Regarding $\theta_{23}$, there is a general preference for non-maximal mixing and the first octant, which is stronger in $\mathrm{NH}$, where the second octant is disfavoured at $\sim 2.6 \sigma$, whereas in IH the two octants are nearly degenerate. In both hierarchies maximal mixing is disfavoured at $2 \sigma$. With respect to the analysis in [2] we find a stronger indication for $\sin ^{2} \theta_{23} \neq 0.5$, which is related to the latest disappearance data of NOvA. In contrast to what reported by $\mathrm{T} 2 \mathrm{~K}$ data, which is consistent with a nearly maximal mixing of $\theta_{23}$, NOvA alone disfavours $\sin ^{2} \theta_{23}=0.5$ at $2.5 \sigma$.

\section{Parameter covariances}

In this section we show and interpret the joint $N \sigma$ contours (covariances) for selected pairs of oscillation parameters. Figure 2a shows the interplay of the CP-violating phase $\delta$ with the mixing parameters $\sin ^{2} \theta_{13}$, where all other parameters have been marginalized away. The top (bottom) row 
refers to the case of normal (inverted) hierarchy. From left to right, the panels refer to increasingly rich data sets. The leftmost panels show the wavy bands allowed by LBL Acc.+Solar+KL data, with a bumpy structure due to the octant ambiguity and the anticorrelation between $\sin ^{2} \theta_{23}$ and $\sin ^{2} \theta_{13}$. In the middle panels, SBL Reactor data select a narrow vertical band, which does not alter significantly the preference for $\delta \simeq 3 \pi / 2$ already present in LBL Acc.+Solar+KL data alone. Therefore, current bounds on $\delta$ are not the result of a tension between LBL Acc. and SBL Reactor data sets, which on the contrary are highly consistent, but they arise from their synergy. The inclusion of atmospheric neutrino data corroborates the previous indications for $\delta$, with a shifted best fit value $\delta \simeq 1.3-1.4 \pi$ and a reduction of the allowed ranges at 1 and $2 \sigma$ (mainly for $\mathrm{NH}$ ).

Figure. $2 \mathrm{~b}$ displays the 1,2 , and $3 \sigma$ contours in the plane $\left(\sin ^{2} \theta_{23}, \Delta m^{2}\right)$. The octant instability discussed in the previous section emerges at a glance. It is important to note that the less maximal $\theta_{23}$, the larger the uncertainty on $\Delta m^{2}$. This feature is even more pronounced with respect to what obtained in [2], because of the preference for non-maximal mixing in NOvA data set. In this context, future constraints on $\Delta m^{2}$ from SBL reactors might play an important role, because of their independence from $\theta_{23}$. Moreover, their increased precision in the measurement of $\Delta m^{2}$ might give additional information on the mass hierarchy.

\section{Conclusions}

In this contribution we presented an update of the global analysis of neutrino oscillation experiments, based on the latest data sets available. We confirm the preference for $\delta \simeq 3 \pi / 2$ and for $\sin \delta<0$, while the value $\delta=\pi / 2$ is now disfavoured at more than $3 \sigma$. The main result of this analysis is the indication for CP violation in the lepton sector at $90 \%$ confidence level, since both $\delta=\pi$ and $2 \pi$ are disfavoured at $\sim 1.6 \sigma$. Regarding $\theta_{23}$, we find a general preference for non-maximal mixing at $\sim 2 \sigma$, which stems mainly from NOvA data set, but the octant degeneracy remains unresolved, since both octants are allowed at $3 \sigma$ in both hierarchies. There is currently a $\sim 2 \sigma$ indication in favour of normal hierarchy, which arises consistently from both LBL Acc. and atmospheric data.

\section{References}

[1] C. Patrignani et al. (Particle Data Group), Chin. Phys. C, 40, 100001 (2016).

[2] F. Capozzi, E. Lisi, A. Marrone, D. Montanino and A. Palazzo, Nucl. Phys. B 908 (2016) 218.

[3] K. Iwamoto, talk at ICHEP 2016, International Conference on High Energy Physics (Chicago, United States, 2016).

[4] P. Vahle, talk at Neutrino 2016, International Conference on Neutrino Physics and Astrophysics (London, England, 2016).

[5] Z. Yu, talk at Neutrino 2016, International Conference on Neutrino Physics and Astrophysics (London, England, 2016).

[6] Kyung Kwang Joo, talk at Neutrino 2016, International Conference on Neutrino Physics and Astrophysics (London, England, 2016).

[7] S. Moriyama, talk at Neutrino 2016, International Conference on Neutrino Physics and Astrophysics (London, England, 2016). 